\title{
Biomasa y calidad nutricional de cuatro variedades de alfalfa para introducir en Pamplona, Colombia
}

\section{Biomass and nutritional quality of four varieties of alfalfa to be introduced in Pamplona, Colombia}

Fecha de recepción: 7 de julio de 2017

Fecha de aprobación: 12 de diciembre de 2017
Alfonso Eugenio Capacho-Mogollón ${ }^{1}$ Dixon Fabián Flórez-Delgado ${ }^{2}$ Johan Fernando Hoyos-Patiño ${ }^{3}$

DOI: http://doi.org/10.19053/01228420.v15.n1.2018.7757

\section{Resumen}

La investigación se desarrolló en el Centro de Investigación en Sanidad Vegetal y Bioinsumos de la Universidad de Pamplona (Norte de Santander), con el objetivo de evaluar la productividad y la calidad nutricional de cuatro variedades de alfalfa (Medicago sativa L.): Cuf 101, Moapa 69, SW-8210 y SW-8718. Se adelantó un experimento con cuatro tratamientos aleatorizados con tres repeticiones, para un total de 12 unidades experimentales de $9 \mathrm{~m}^{2}$ cada una. La aplicación de correctivos y enmiendas se realizó una semana antes de la siembra, con base en el análisis de suelos. Al día 70 de la siembra se realizó un corte de emparejamiento para homogeneizar cada parcela. Las mediciones de producción de biomasa verde y calidad nutricional se determinaron en el estadio de botón floral, a los 210 días de la siembra. Se aplicaron pruebas de análisis de varianza ANOVA, no encontrándose diferencias significativas entre las variedades de alfalfa para las condiciones ambientales del municipio de Pamplona, por lo que todas pueden introducirse en este agroecosistema.

Palabras clave: digestibilidad in vitro; forraje; leguminosa; Medicago sativa.

\begin{abstract}
This study was carried out at the Center of Research in Plant Health and Bioinsumes of the University of Pamplona (Norte de Santander), with the objective of evaluating the productivity and nutritional quality of four alfalfa (Medicago sativa L.) varieties: Cuf 101, Moapa 69, SW-8210, and SW-8718. An experiment was carried out with four randomized treatments with three replicates, for a total of 12 experimental units

1 M. Sc. Universidad de Pamplona (Pamplona, Colombia). aecapacho@unipamplona.edu.co.

2 M. Sc. Universidad de Pamplona (Pamplona, Colombia). dixon.florez@unipamplona.edu.co. ORCID: 0000-0002-3915-8396.

3 M. Sc. Universidad de Pamplona (Pamplona, Colombia). zootecnia@unipamplona.edu.co.
\end{abstract}


of $9 \mathrm{~m}^{2}$ each. The application of correctives and amendments was done one-week prior planting, based on soil analysis. At day 70 of the sowing, a pairing cut was made to homogenize each plot. Measurements of green biomass production and nutritional quality were determined at the floral bud stage, at 210 days after sowing. The ANOVA tests showed no significant differences among the alfalfa varieties for the environmental conditions of the municipality of Pamplona, which means that all varieties can be introduced in this agroecosystem.

Keywords: forage; in vitro digestibility; legume; Medicago sativa.

\section{Para citar este artículo:}

Capacho-Mogollón AE., Flórez-Delgado DF., Hoyos-Patiño JF. Biomasa y calidad nutricional de cuatro variedades de alfalfa para introducir en Pamplona, Colombia. Ciencia y Agricultura. 2018; 15(1): 61-67. 


\section{Introducción}

En América Latina y el Caribe, la actividad ganadera ocupa la mayor proporción de uso del suelo (1), y su principal característica es la baja productividad y rentabilidad. González (2) menciona que debido a la expansión de la frontera ganadera se están generando efectos negativos en los ecosistemas terrestres, con actividades como tala y quema de bosques, erosión de suelos y compactación (3), pérdida de la biodiversidad vegetal (4), al igual que contaminación de aguas por uso de agroquímicos (5).

En Colombia, de acuerdo con lo reportado en los resultados de la Encuesta Nacional Agropecuaria (6), el área total de uso del suelo es de 114.174.800 ha; el $45 \%$ de estas (50.941.744 ha) están dedicadas a las actividades del sector agropecuario, y el $77 \%$ (39.196.059 ha), a la actividad pecuaria. La producción de pastos y de forrajes se desarrolla en 39.196.059 ha (el $81 \%-31.631 .064$ ha- se encuentran cultivadas en pastos, y el restante $19 \%$ -7.564.996 ha- estarían cubiertas de malezas y rastrojos (7).

En la provincia de Pamplona existe una población de 456.026 bovinos, que pastorean en 466.833 ha (8), con una capacidad de carga de 0.69 Unidad Animal (U.A.) y con una producción promedio de leche de $3.6 \mathrm{~kg}$ animal/día (9). Los análisis de la información realizados por el Centro de Información Tecnológica Digital (CITD), de la Facultad de Ciencias Agrarias de la Universidad de Pamplona, cofinanciados por el Departamento Administrativo de Ciencia, Tecnología e Innovación (10), señalan que en la Provincia de Pamplona existen 125.154 ha dedicadas a la producción agropecuaria, una población bovina de 67.125 animales, 12.346 vacas en ordeño, con una producción de $3.9 \mathrm{~kg}$ leche por vaca por día, y una capacidad de carga 0,54 animales por ha. Estos indicadores señalan una baja competitividad de los sistemas de producción pecuarios de la región, en relación con los indicadores de los demás departamentos del país, en los que se registra en promedio 0,69 animales por ha (6).
Uno de los principales aspectos que causan estos bajos indicadores se relaciona con el mal manejo de las praderas, el uso de forrajes tradicionales y el desconocimiento de especies forrajeras adaptadas a la zona (11). En la provincia de Pamplona (Norte de Santander) se manejan gramíneas tradicionales, como kikuyo (Pennisetum clandestinum $\mathrm{H}$.), raigrass (Lolium sp.) y falsa poa (Holcus lanatus L.), de baja calidad nutricional en relación con otros forrajes como la alfalfa (Medicago sativa L.), que, además de adecuados aportes nutricionales para los rumiantes, tiene una alta capacidad de adaptación a condiciones climáticas adversas (12).

La situación anteriormente presentada condiciona el mantenimiento y producción de los animales a un necesario uso de concentrados comerciales, el cual incrementa de manera notable los costos de inversión, produciendo, por tanto, menores ganancias netas para los productores de la zona (13); ante ello resulta necesario utilizar material forrajero adecuadamente adaptado a las condiciones geográficas y ambientales de la región, pero que además satisfaga los requerimientos nutricionales de los animales, y así disminuir el uso de suplementos alimenticios.

La presente investigación abordó la problemática planteada a partir de cuatro variedades de alfalfa (M. sativa), en busca de una alternativa forrajera adaptada a las condiciones ambientales del municipio de Pamplona, y viable para los productores de la región.

\section{Materiales y métodos}

\section{A. Localización}

La investigación se desarrolló en el Centro de Investigación en Sanidad Vegetal y Bioinsumos CISVEB-, de la Universidad de Pamplona, ubicado en la zona norte del municipio de Pamplona, a una altitud de 2287 m s.n.m., con una temperatura promedio de $14{ }^{\circ} \mathrm{C}$. El suelo es de textura francoarenosa, con mediana capacidad de retención de humedad y nutrientes, buen drenaje y aireación y $\mathrm{pH}$ fuertemente ácido (14). 


\section{B. Material vegetal}

Para esta investigación se definieron las siguientes variedades de alfalfa (M. sativa): Cuf 101, Moapa 69, SW-8210 y SW-8718, debido a que presentan crecimiento erecto y rápida recuperación, con una producción promedio de 60-80 toneladas de forraje verde año, soportando una capacidad de carga de 3 a 4 animales (15), en busca de introducir aquellas variedades con mejor comportamiento en el municipio de Pamplona.

\section{Diseño experimental}

Se usó un diseño de bloques completos aleatorizados (teniendo como criterio de bloqueo el terreno), con cuatro variedades y tres réplicas, para un total de 12 unidades experimentales de $9 \mathrm{~m}^{2}$ cada una.

\section{Procedimiento experimental}

La investigación inició con la identificación de las propiedades físicas y químicas del suelo; para ello, se tomó una muestra de suelo y se remitió al laboratorio SIAMA Ltda., en la ciudad de Bucaramanga para su análisis. La siembra se realizó a razón de $25 \mathrm{~kg}$ de semilla por hectárea (14) en surcos separados por $25 \mathrm{~cm}$ y siembra a chorrillo (15).

Una semana antes de la siembra se aplicaron los correctivos y enmiendas, incorporados en los 10 primeros centímetros del suelo, según lo referencia Heredia A. (16), tomando como base los resultados y recomendaciones del análisis de suelos, hasta alcanzar los requerimientos nutricionales de la alfalfa.

\section{E. Toma de datos}

1) Producción de biomasa. El forraje verde se midió en función de su peso; para ello se tomó una muestra de $1 \mathrm{~m}^{2}$ de cada parcela a una altura de 5 $\mathrm{cm}$. El resultado que se obtuvo se proyectó al área de la parcela y se estimó la producción por hectárea. En el caso de la materia seca, se obtuvo en el análisis de laboratorio, y, de la misma manera, se hizo la proyección a una hectárea.
2) Análisis bromatológico. Se tomó una muestra de forraje de $1 \mathrm{~kg}$ de cada parcela y se remitió al laboratorio de la Universidad Nacional, en la ciudad de Bogotá, para su procesamiento. Se solicitó análisis de materia seca (MS), humedad $(\mathrm{H})$, proteína (Prot.), fibra detergente ácida (FDA), fibra detergente neutra (FDN), energía (E), calcio (Ca), fósforo (P) y digestibilidad in vitro (DIV).

\section{F. Pruebas estadísticas}

Se aplicaron pruebas de análisis de varianza ANOVA de un factor a través del software estadístico SPSS versión 18. Las medias se compararon por la prueba de Tukey $(\mathrm{P}<0.05)$.

\section{Resultados y discusión}

En la Tabla I se muestran las comparaciones múltiples de las cuatro variedades de alfalfa en sus diferentes factores de estudio (materia seca, proteína, FDN, FDA, cenizas, DIV, calcio, fósforo y producción de biomasa).

Tabla I. Prueba de homogeneidad de varianzas.

\begin{tabular}{|c|c|c|}
\hline & $\begin{array}{l}\text { Estadístico } \\
\text { de Levene }\end{array}$ & Sig.* \\
\hline Porcentaje de materia seca & 1,652 & ,253 \\
\hline Producción de biomasa & 1,557 & ,274 \\
\hline Porcentaje de proteína & 3,401 & 074 \\
\hline Porcentaje de fibra neutra & 1,345 & 327 \\
\hline Porcentaje de fibra ácida &, 535 & 671 \\
\hline Porcentaje de ceniza & 2,733 & 1114 \\
\hline Porcentaje de digestibilidad & 3,855 &, 056 \\
\hline Porcentaje de calcio & ,994 & 443 \\
\hline Porcentaje de fósforo & 2,469 & 136 \\
\hline
\end{tabular}

Se puede observar que las variaciones intergrupos e intragrupos tienen resultados estadísticos similares $(\mathrm{P}>0.05)$.

\section{A. Producción de biomasa}

Se puede evidenciar que no existen diferencias significativas $(p>0.05)$ entre las cuatro variedades alfalfa en cuanto a las medias de producción de 
biomasa $\left(\right.$ t.ha $\left.{ }^{-1}\right)$. La producción de las variedades de alfalfa en esta investigación (5.43 t.ha-1) fue ampliamente inferior, comparada con los datos reportados por Rivera et al. (17) al segundo corte (7.5 t.ha-1) y Martínez D. (18) en el segundo corte $(8,79$ t.ha-1).

En la Tabla II se muestran las medias para producción de forraje verde y contenido de materia seca.

Tabla II. Medias de producción de Forraje Verde y Materia Seca.

\begin{tabular}{|c|c|c|c|c|c|}
\hline $\begin{array}{c}\text { Variedad de } \\
\text { alfalfa }\end{array}$ & $\mathbf{N}^{*}$ & $\begin{array}{c}\text { Forraje } \\
\text { verde } \\
\left(\text { t.ha- }^{-1}\right)\end{array}$ & DS** & $\begin{array}{c}\text { Materia seca } \\
\text { (\%) }\end{array}$ & DS** $^{*}$ \\
\hline Moapa-69 & 3 & 5,11 & 2,42378 & 9,23 & 1,78979 \\
\hline SW-8718 & 3 & 4,81 & 1,44652 & 9,43 & 1,00167 \\
\hline SW-8210 & 3 & 6,40 & 3,86690 & 10,06 & 1,10151 \\
\hline Cuf-101 & 3 & 5,39 & 2,73128 & 10,50 & 0,79373 \\
\hline Promedio & 12 & 5,43 & 2,43225 & 9,80 & 1,17354 \\
\hline
\end{tabular}

*N: número de réplicas. **DS. Desviación Estándar.

\section{B. Materia seca}

Se evidencia que no existen diferencias significativas $(p>0.05)$ entre las cuatro variedades de alfalfa en cuanto a las medias de materia seca. Dammer (19) evaluó cuatro variedades de esta leguminosa, en cuanto a la producción de materia seca por hectárea, al segundo corte, en época de invierno, y obtuvo 3869 kg.ha-1 MS en la variedad Moapa 69; seguida por la Cuf 101, con una producción de 3319,7 kg.ha-1 MS, y, por último, la SW-8210, con $2393 \mathrm{~kg}$.ha-1 MS. Se puede constatar que la investigación de Dammer arrojó unos promedios por debajo de los de la presente investigación en las mismas variedades. Los datos aportados por Plevich, Delgado \& Saroff (20) en materia seca, para segundo corte, fueron de $2820 \mathrm{~kg} \cdot \mathrm{ha}^{-1}$. Maekawa \& Demateis (21) reportan una produccion al segundo corte de $1770 \mathrm{~kg}$.ha-1 de materia seca. Morales (22) reporta en su trabajo una produccion de $4160 \mathrm{~kg} \cdot \mathrm{ha}^{-1}$ al segundo corte.

\section{Calidad nutricional}

La calidad nutricional de cada variedad de alfalfa se ofrece en la Tabla III. No se presentó diferencia estadística entre los tratamientos para ninguna de las variables.

Tabla III. Medias de la calidad nutricional de cada pasto.

\begin{tabular}{|l|c|c|c|c|c|c|c|c|c|c|c|c|c|c||}
\hline & $\begin{array}{c}\text { Proteína } \\
(\mathbf{\%})\end{array}$ & DS & $\begin{array}{c}\text { FDN } \\
(\mathbf{\%})\end{array}$ & DS & $\begin{array}{c}\text { FDA } \\
\mathbf{( \% )}\end{array}$ & DS & $\begin{array}{c}\text { Ceniza } \\
(\mathbf{\%})\end{array}$ & DS & $\begin{array}{c}\text { Ca } \\
\mathbf{( \% )}\end{array}$ & DS & $\begin{array}{c}\text { P } \\
(\mathbf{\%})\end{array}$ & DS & $\begin{array}{c}\text { DIV } \\
(\mathbf{\%})\end{array}$ & DS \\
\hline Moapa-69 & 21,10 & 0,51 & 49,50 & 4,33 & 38,56 & 4,77 & 17,26 & 5,97 & 1,473 & 0,17 & 0,36 & 0,05 & 50,26 & 7,33 \\
\hline SW-8718 & 20,40 & 3,01 & 45,80 & 5,45 & 33,53 & 4,50 & 13,20 & 3,12 & 1,66 & 0,32 & 0,34 & 0,02 & 52,76 & 6,62 \\
\hline SW-8210 & 18,83 & 0,51 & 53,63 & 2,54 & 40,56 & 2,84 & 14,43 & 1,48 & 1,59 & 0,15 & 0,37 & 0,01 & 49,90 & 0,60 \\
\hline Cuf-101 & 19,76 & 2,59 & 52,30 & 3,37 & 38,06 & 4,33 & 14,80 & 3,14 & 1,38 & 0,20 & 0,40 & 0,02 & 48,60 & 3,12 \\
\hline Promedio & 20,02 & 1,93 & 50,30 & 4,67 & 37,68 & 4,46 & 14,92 & 3,58 & 1,52 & 0,22 & 0,37 & 0,03 & 50,38 & 4,70 \\
\hline
\end{tabular}

FDN: Fibra Detergente Neutra; FDA: Fibra Detergente Ácida; Ca: Calcio; P: Fósforo; DIV: Digestibilidad in vitro; DS: Desviación Estándar.

\section{Proteína}

La proteína varió entre el $18,8 \%$ y el $21,1 \%$, con una media de $20,02 \%$; este porcentaje no es tan inferior a los reportados por García et al. (23): 23,89\%, y Basigalup (24): 23,7\%, en el estadio de botón floral realizado en el segundo corte de proteina. Los resultados obtenidos se ajustan a lo reportado por Plevich, Delgado \& Saroff (20), de $20 \%$ de proteína en segundo corte. 


\section{E. Fibra detergente neutra}

Se obtuvo un rango de medias en fibra detergente neutra que osciló entre el $45 \%$ y el $54 \%$. González (25) informa en su trabajo un $51 \%$ de FDN, mientras que Basigalup (24) refiere un $32,7 \%$ de FDN en segundo corte en el estadio de botón floral.

\section{F. Fibra detergente ácida}

La FDA de las cuatro variedades de alfalfa varió entre 33,53 \% y 40,56 \%. López (26) reportó 30,0 $\%$, y Cardona (28), 29,9\%, también inferiores a los de la presente investigación.

\section{G. Cenizas}

El porcentaje de cenizas en la presente investigación varió entre el 13,20 y el 17,26. López (26) informó que obtuvo un 2,36 \% de ceniza, mientras que Puente, Soto \& Ramírez (35) reportaron datos en un rango del $10 \%$ al $12 \%$, siendo muy inferior a lo reportado en esta investigación.

\section{$H$. Digestibilidad in vitro}

El porcentaje de digestibilidad in vitro en la presente investigación varió entre el 48,60 y el 52,76 , inferior al 63,88 \% reportado por Delgado (28) en la fase fenológica de botón; al 60,67 \% encontrado por Meza et al. (29) en segundo corte, y al 71,7\% obtenido por Arreaza y Sánchez (30) al segundo corte.

\section{Calcio}

Se obtuvo en esta investigación un rango entre el $1.3 \%$ y el $1.7 \%$ para parámetro nutricional calcio (\%), semejante al encontrado por Mora (31): $1,36 \%$, y por Mufarrege (32), quien halló un porcentaje de calcio del 1,43\%. Rodríguez et al. (33) reportan un $0.56 \%$ de calcio, valor por debajo de los reportados en el presente trabajo.

\section{J. Fósforo}

Entre las cuatro variedades de alfalfa no se evidencian diferencias de las medias en cuanto al por- centaje de fósforo. Mora (31) indica un porcentaje del $0,27 \%$ de fósforo, ligeramente más bajo que en la presente investigación, y se encuentra en los parámetros adecuados de concentración. Urbano y Dávila (34) mencionan el 0,37 \% de fósforo en su investigación al segundo corte. Mufarrege (32) obtuvo un porcentaje de fósforo del $0,38 \%$ en alfalfa, el cual se asemeja a los obtenidos en este trabajo.

\section{Conclusiones}

La presente investigación concluye que es factible establecer en la región de Pamplona las cuatro variedades de alfalfa evaluadas (Medicago sativa L.), dado que las condiciones agroecológicas de la zona permiten su desarrollo. Entre las variedades de alfalfa estudiadas bajo las condiciones ambientales del CISVEB de la Universidad de Pamplona no existen diferencias estadísticas significativas en cuanto a la producción de biomasa y los valores de los parámetros nutricionales: biomasa, materia seca, proteína, calcio, fósforo y digestibilidad in vitro; valores que se ubicaron dentro de los parámetros normales contemplados en la literatura.

El crecimiento y desarrollo de las cuatro variedades de alfalfa (Medicago sativa L.) indican la capacidad de estas para establecerse a este medio agroecológico; esta adaptación está dada por la disponibilidad de yemas (tejido meristemático activo) y la reserva y movilización de carbohidratos solubles, que favorecen el proceso de fotosíntesis y, por tanto, el crecimiento de nuevos tallos, hojas, rebrotes y ramas primarias.

Es importante mencionar que es necesario seguir realizando estudios similares y complementar con investigaciones que evalúen el desempeño de los animales que cuenten con esta leguminosa como parte de su dieta.

\section{Referencias}

(1) Food and Agriculture Organization (FAO). Ayudando a desarrollar una ganadería sustentable en Latinoamérica y el Caribe: lecciones a partir de casos exitosos. Santiago de Chile. 101 p. 2008.

(2) González S. Impacto ambiental de la ganadería. INIA. La Platina. Chile. 41 p. 2010. 
(3) Universidad de Buenos Aires (UBA). Las plantas y los minerales. Guía de nutrición mineral. Argentina. 2009.

(4) Fernández E. Metodologías para la evaluación y mejora del impacto ambiental de los sistemas ganaderos: análisis comparado y posibilidades de aplicación en el sector de los pequeños rumiantes de Andalucía. Tesis de Maestría. Universidad de Córdoba, Argentina. 2009.

(5) Food and Agriculture Organization (FAO). Las repercusiones del ganado en el medioambiente. El desafío estriba en reconciliar dos demandas: la de productos animales y la de servicios ambientales. (En línea). 2006. (Fecha de acceso: 2 de marzo del 2015), disponible en: http://www.fao.org/ag/esp/revista/0612sp1.htm. 2006.

(6) Encuesta Nacional Agropecuaria (en línea). Disponible en: http:// goo.gl/Fi11Yq. 2009.

(7) Bernal L., Suárez R. La producción de forraje en el contexto del cambio climático. | Bogotá-Colombia. Rev. Cienc. Anim. 2011; 4: 7-14.

(8) FEDEGAN. Análisis del inventario ganadero colombiano. Comportamiento y variables explicativas. 2013.

(9) López H. Diseño del producto turístico de Norte de Santander. Fondo Nacional de Turismo FONTUR. Bogotá, D.C. 2014.

(10) Centro de Información Tecnológico Digital CITD. Caracterización de los sistemas de producción de la provincia de Pamplona. Universidad de Pamplona. 2008.

(11) Castaño J. Adaptación y manejo de especies forrajeras y técnicas para optimizar su producción. EEA INTA Balcarce. Argentina. 2003.

(12) Hernández D., Flórez D., Villamizar C., Capacho A. Materiales promisorios para la producción de pastos en el trópico alto de la provincia de Pamplona. Universidad de Pamplona. 2010.

(13) Stehr W. Alimentos complementarios para producción de carne. Cenerema, UACH. 2001.

(14) Ministerio de Agricultura y Desarrollo Rural, Departamento Administrativo Nacional de Estadística (DANE), Federación Nacional de Arroceros (Fedearroz). Distrito de Riego USOCOELLO, Aspectos agrícolas y pecuarios. 2001.

(15) Cabrera P. Producción de siete cultivares de alfalfa (Medicago sativa L.) en un andisol de la región de la Araucanía. Universidad de la Frontera. Temuco, Chile. 2004.

(16) Heredia A. Evaluación del comportamiento forrajero del Medicago sativa bajo la aplicación de diferentes niveles de micorrizas y abono orgánico bovino. Facultad de Ciencias Pecuarias. Escuela Superior Politécnica de Chimborazo, Riobamba, Ecuador. 2011.

(17) Rivera M., Martínez JG., Sánchez I. Tecnología de producción de alfalfa (Medicago sativa) mediante riego por goteo subsuperficial o subterráneo. Agrofaz. 2005; 5(3): 23-28.

(18) Martínez D. Nutrición y alimentación animal. Valoración energética de los alimentos. Departamento de Producción Animal, Universidad de Córdoba, Argentina. 2011.

(19) Dammer M. Adaptación de cuatro variedades de Alfalfa Medicago Sativa en la zona de Cananvalle-Tabacundo. Cayambe-Ecuador. 2014.

(20) Plevich J., Delgado A., Saroff C. El cultivo de alfalfa utilizando agua de perforación, agua residual urbana y precipitaciones. Rev. bras. eng. agríc. ambient. 2012; 16(12). DOI: http://doi.org/10.1590/ S1415-43662012001200013.

(21) Maekawa M., Demateis F. Producción de biomasa de pasturas de alfalfa. Instituto Nacional de Tecnología Agropecuaria, Argentina. 2013.

(22) Morales J., Jiménez J., Velasco A., Villegas Y., Enríquez del Valle J., Hernández A. Evaluación de 14 variedades de alfalfa con fertirriego en la Mixteca de Oaxaca. Téc Pecu Méx. 2006; 44(3): 277-288.

(23) García C., Vázquez B., Lorenzo L., García A., Petisco' C., Vicente S., García Y. Evaluación de 26 cultivares de alfalfa en el Oeste Español. Pastos. 2010; 40(2): 189- 210.

(24) Basigalup D. El cultivo de la alfalfa en la Argentina. EEA Manfredi-INTA. Argentina. 2004.

(25) González R. Ensilaje de alfalfa (Medicago sativa L.) y ovillo (Dactylis glomerata L.) en contenedores de 200 litros durante las épocas de lluvia. Universidad Autónoma de Chapingo. Chapingo, Estado de México.

(26) López A. Evaluación de diferentes niveles de vinaza aplicados basalmente en la producción forrajera de Medicago sativa (alfalfa). Escuela Superior Politécnica de Chimborazo. Riobamba Ecuador. 2011.

(27) Cardona E., Ríos L., Pena J. Disponibilidad de variedades de pastos y forrajes como potenciales materiales lignocelulósicos para la producción de bioetanol en Colombia. Inf. Tecnol. 2012; 23(6).

(28) Delgado E. La alfalfa. Estudio comparativo de variedades comercializadas en España. Semillas y Cultivos. 2005.

(29) Meza G., Sánchez A., Meza M., Meza C., Franco N., Avellaneda J., Estupiñán K., Barrera A., Cabrera R., Vera D., Liuba G. Digestibilidad in vivo de forrajeras arbustivas tropicales para la alimentación de cuyes (Cavia porcellus Linnaeus), en el litoral ecuatoriano. Veterinaria y Zootecnia. 2012; 6(2)

(30) Arreaza L., Sánchez D., Abadía B. Degradabilidad ruminal de fracciones de carbohidratos en forrajes tropicales determinada por métodos in vitro e in situ. Revista Corpoica. 2005; 6(1): 51-58. DOI: http://doi.org/10.21930/rcta.vol6_num1_art:37.

(31) Mora J. Adaptación de ocho variedades comerciales de alfalfa (Medicago sativa) sobre los 2900 m s.n.m. en el sector de pailones en la hacienda El Prado. Ecuador. 2005.

(32) Mufarrege, Q. El calcio en la alimentación del ganado bovino para carne. E.E.A. INTA Mercedes, Corrientes. Argentina. 2002.

(33) Rodríguez M., González A., Yáñez A., Silva M., Gómez C. Composición química de recursos forrajeros para la alimentación de ovinos en Colima. Centro de Investigación Regional Pacífico Centro. México. Folleto técnico Núm. 3. 2013.

(34) Urbano D., Dávila C. Evaluación del rendimiento y composición química de once variedades de alfalfa (Medicago sativa) bajo corte en la zona alta del estado Mérida, Venezuela. Rev. Fac. Agron. 2003; 20(1): 97-107.

(35) Puente J., Soto V., Ramírez S. Determinación de calidad forrajera y su índice de producción de leche de seis variedades de alfalfa (Medicago sativa L.) evaluados en la comarca lagunera en el 2001 y 2002. Universidad Agraria Antonio Nariño, México. 2002. 
